\title{
Docencia de la asignatura Interacción Persona-Ordenador en tiempos de pandemia: una experiencia con Microsoft Teams Teaching Human-Computer Interaction in pandemic time: an experience with Microsoft Teams
}

\author{
Roberto Therón ${ }^{1}$, Alicia García-Holgado ${ }^{1}$, Samuel Marcos-Pablos ${ }^{1}$ \\ theron@usal.es, aliciagh@usal.es, samuelmp@usal.es \\ ${ }^{1}$ Dpto. Informática y Automática, \\ Grupo de Investigación GRIAL \\ Universidad de Salamanca \\ Salamanca, España
}

\begin{abstract}
Resumen- Todos los sectores de la sociedad se han visto afectados durante la crisis sanitaria causada por la COVID-19. La pandemia ha impulsado cambios en el sector educativo, principalmente relacionados con la transformación digital de los procesos. Estos cambios no solo en el uso de la tecnología sino también en el enfoque metodológico utilizado. En este escenario, se desarrolla la adaptación de la asignatura Interacción Persona-Ordenador, primero a formato online durante el curso 2019-20, y después a formato presencial con retransmisión online para el curso 2020-21. En este proceso, la incorporación de Microsoft Teams ha permitido reforzar la metodología de aprendizaje basado en proyectos y mejorar la comunicación con el alumnado. Este trabajo describe la experiencia como ejemplo para incorporar Microsoft Teams en otras asignaturas independientemente de si la docencia se desarrolla en formato presencial, online o híbrido.
\end{abstract}

Palabras clave: interacción persona-ordenador, COVID-19, elearning, educación superior, tutorías

\begin{abstract}
The health crisis caused by COVID-19 has affected all sectors of society. The pandemic has driven changes in the education sector, mainly related to the digital transformation of processes. These changes are not only in the use of technology but also in the methodological approach used. In this context, the course HumanComputer Interaction was adapted, first to an online format during the 2019-20 academic year, and then to a face-to-face format with online streaming for the 2020-21 academic year. During this process, the integration of Microsoft Teams has reinforced the project-based learning methodology and improved communication with the students. This paper describes the experience as an example for including Microsoft Teams in other courses, regardless of whether the teaching takes place in face-to-face, online or hybrid format.
\end{abstract}

Keywords: human-computer interaction, COVID-19, elearning, higher education, tutoring

\section{INTRODUCCIÓN}

La pandemia causada por el virus SARS-CoV-2 ha tenido un claro impacto en todos los sectores de la sociedad a nivel mundial. En el contexto educativo, ha implicado diferentes medidas que abarcan desde el cierre de colegios y universidades (Zubillaga \& Gortazar, 2020) hasta la completa transformación de la docencia marcada principalmente por una alta presencia de evaluación no presencial (Abella García et al., 2020; Fardoun et al., 2020; García-Peñalvo et al., 2020).

Según el análisis realizado por García-Peñalvo, Corell, et al. (2021), en el ámbito universitario, el impacto de la pandemia ha servido para acelerar la transformación digital de los procesos, con énfasis en el área docente, generando un cambio en la comunidad universitaria en cuanto a la aceptación de la tecnología presente en los procesos.

Es indudable que el uso de las tecnologías de la información y la comunicación (TIC) se ha incrementado durante la pandemia como herramientas para cubrir las nuevas necesidades tanto de docentes como de los propios estudiantes. El uso de las TIC en el proceso educativo debe ir siempre acompañado de un enfoque metodológico adecuado, aunque se ha detectado un preocupante desfase en el uso y aplicación de metodologías docentes en el aula (García-Peñalvo \& Corell, 2020).

En este escenario, se desarrolla la adaptación de la asignatura Interacción Persona-Ordenador impartida en el segundo cuatrimestre del tercer curso del Grado en Ingeniería Informática de la Universidad de Salamanca. Esta asignatura se centra en ofrecer los fundamentos básicos de Interacción Persona-Ordenador de tal forma que introduce al alumnado la importancia del factor humano en el diseño de interfaces de usuario.

El objetivo de este trabajo es ilustrar cómo la incorporación de Microsoft Teams ha permitido reforzar la metodología de aprendizaje basado en proyectos (Estruch \& Silva, 2006; Macias, 2012) durante la transformación de la docencia debido a las medidas implantadas en la Universidad de Salamanca por la pandemia. En particular, se describe la integración de la herramienta en los mecanismos de aprendizaje con docencia presencial.

El trabajo se ha organizado en cinco apartados. El segundo apartado presenta el enfoque de la asignatura previo a la transformación realizada durante el curso 2019-20. El tercer apartado presenta la integración de Microsoft Teams en el proceso de enseñanza-aprendizaje. El cuarto apartado plantea los principales resultados de la experiencia. Finalmente, el 
último apartado contiene la discusión y las principales conclusiones del trabajo.

\section{CONTEXTO}

El 13 de marzo de 2020 se llevó a cabo la suspensión de las clases presenciales y la imposibilidad de acceder a los edificios de la Universidad de Salamanca como parte de las medidas para gestionar la crisis sanitaria. Este cambio implicó dos grandes fases, una primera marcada por la emergencia de dar respuesta casi inmediata, sin tener tiempo de planificar y rediseñar las asignaturas (Llorens-Largo, 2020), y una segunda fase marcada por la decisión de realizar una evaluación no presencial y finalizar el curso académico a distancia.

Los equipos docentes se ven abocados a adaptar la docencia presencial a formato totalmente no presencial con los recursos tecnológicos disponibles en la institución. Aunque esta situación puso en evidencia ciertas brechas tecnológicas y metodológicas (García-Peñalvo, Corell, et al., 2021), también existen casos de éxito tales como (García-Peñalvo, GarcíaHolgado, et al., 2021; Hernández-Ramos et al., 2021; LlorensLargo et al., 2021).

En el caso de la asignatura Interacción Persona-Ordenador del Grado de Ingeniería Informática de la Universidad de Salamanca la adaptación a la docencia no presencial ha sido abordada partiendo de la programación original de las clases presenciales en las que ya se aplicaban un conjunto de metodologías activas, principalmente el aprendizaje basado en proyectos. El trabajo se organiza por temas y prácticas entregables.

El principal elemento de comunicación fue el Campus Virtual de la Universidad de Salamanca, Studium. Esta herramienta de aprendizaje ya se utilizaba en cursos previos para gestionar los contenidos, la entrega de prácticas y la coordinación con los estudiantes aunque con una baja o nula interacción de los mismos.

La interacción con el alumnado se completa con el uso de redes sociales, concretamente Twitter, donde el profesorado comparte contenidos complementarios a los vistos en las sesiones presenciales, así como noticias de actualidad relacionadas con los temas tratados en la asignatura. El uso de la red social se mantuvo durante la transformación en docencia online aunque se es consciente que no todo el alumnado dispone de cuenta o utiliza Twitter.

Finalmente, el soporte tecnológico de la asignatura se completa durante el periodo online con Blackboard Collaborate, herramienta de videoconferencia integrada con el campus virtual. Esta permitió la realización de sesiones síncronas que reemplazaron a las sesiones presenciales. Para evitar la sobrecarga de sesiones síncronas y respetar los horarios previstos en la docencia presencial se plantearon cumpliendo las siguientes premisas: (1) las sesiones síncronas tenían una duración menor que las sesiones presenciales; (2) las sesiones síncronas se grababan y se facilitaba el vínculo a los estudiantes a través del campus virtual para su consulta asíncrona en el momento que se adapte a sus necesidades; (3) se estableció un calendario para evitar repetir sesiones síncronas y dar más flexibilidad al alumnado, de tal forma que las sesiones correspondientes a teoría se impartían en semanas alternas a un grupo de estudiantes (Grupo TA o Grupo TB) y las sesiones de prácticas también se alternaban entre los tres grupos de prácticas (P1, P2 o P3).

Además, se elaboraron píldoras de vídeo con explicaciones de herramientas necesarias para que los estudiantes desarrollen las entregas de prácticas y la práctica final. Estos vídeos permitían reducir el número de sesiones síncronas a la par que ofrecer materiales más condensados y adaptados a las necesidades del alumnado. Todas las píldoras se publicaron en YouTube en abierto.

Finalmente, durante el periodo online el alumnado comenzó a solicitar tutorías, de tal forma que se tomó la decisión de incorporar una herramienta que facilitara la comunicación con el alumnado a la par que permitir la gestión de tutorías de forma más dinámica. Por este motivo se comienza a utilizar Microsoft Teams. Se trata de una herramienta de gestión de equipos que está disponible con la cuenta institucional. Se debe matizar que esta herramienta no sustituye al campus virtual tradicional aunque se asemeja más a las herramientas de mensajería que el alumnado está acostumbrado a utilizar, tales como Whatsapp, Telegram o Discord.

El proceso de integración de Microsoft Teams supuso una migración de los principales contenidos disponibles en el campus virtual, de tal forma que el equipo de Teams para la asignatura tuviera una estructura coherente con el espacio del campus. Para ello se crearon tantos canales como temas de teoría había en la asignatura, de tal forma que en cada canal se ponían a disposición del alumnado los principales materiales y a la par servía como espacio de interacción para dudas y cuestiones relacionadas con el temario. Además, se habilita un canal para tutorías y prácticas en el que no solo el alumnado comienza a interactuar y plantear dudas, sino que el profesorado comparte aquellas dudas que le llegan por otros canales, principalmente email, y que pueden ser de utilidad para el resto (Figura 1).

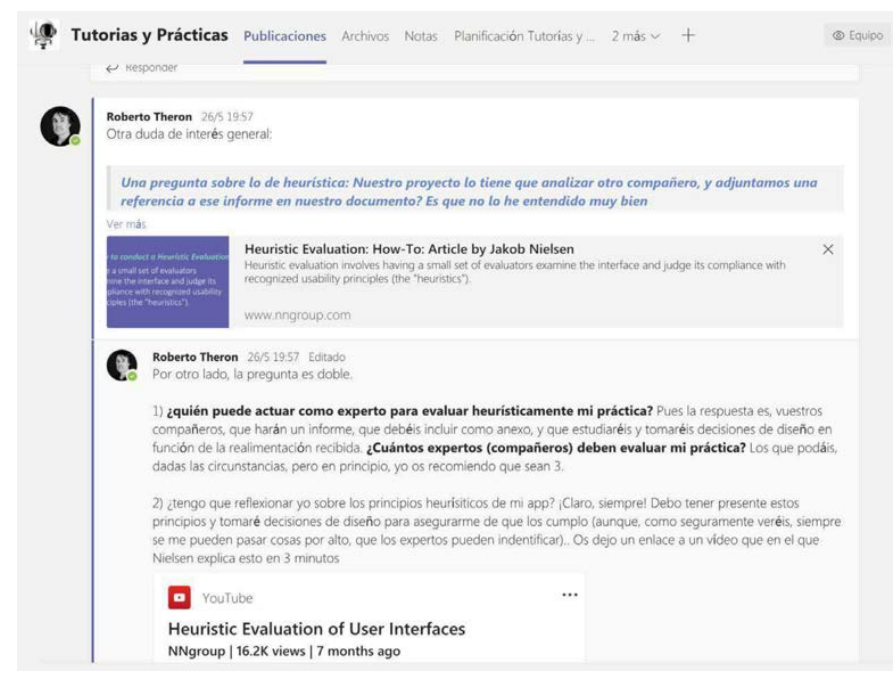

Figura 1. Canal de tutoría y prácticas en el equipo en Microsoft Teams para el curso 2019-20

Asimismo, la integración de Microsoft Teams permite dar solución a otra necesidad que emerge a la hora de transformar ciertas dinámicas de presencial a online. Concretamente una de las prácticas de la asignatura que continúa el trabajo iniciado en el proyecto de innovación "Interfaces imaginadas" iniciado durante el curso 2016-17 (Therón et al., 2017), cuya dinámica central radica en el análisis de películas o series en las que 
aparecen interfaces (reales y/o imaginas). Esta dinámica se realizaba en grupos de forma presencial, de tal forma que cada grupo de prácticas presenta su análisis y posteriormente se genera un debate sobre las interfaces que aparecen en la película o serie. Para adaptar esta dinámica al contexto online, el grupo de estudiantes elabora un vídeo con el análisis realizado, ese vídeo es subido a Microsoft Stream que permite también integrarlo en el equipo de Microsoft Teams, y el debate se desarrolla en una sesión síncrona en Blackboard Collaborate. Además, para asegurar el dinamismo de la sesión al final de cada vídeo dentro de Microsoft Stream se integra un formulario de Microsoft Forms para que todos los participantes reflexionen sobre cuestiones particulares de interacción persona-ordenador.

\section{DESCRIPCIÓN}

Tras la experiencia desarrollada en un contexto de emergencia, se plantea un nuevo escenario durante el curso 2020-21. La Universidad de Salamanca, siguiendo las pautas del Ministerio, plantea como enfoque el modelo híbrido rotatorio (García-Peñalvo, 2020), es decir, la impartición de las clases en formato presencial con retransmisión online para una parte del alumnado que intercala asistencia online y presencial.

Los equipos docentes deben realizar una nueva adaptación, ya que ni el enfoque presencial previo a la pandemia ni el enfoque online del confinamiento encajan en el modelo híbrido rotatorio.

El segundo proceso de adaptación de la asignatura Interacción Persona-Ordenador se sustenta en dos decisiones fundamentales. En primer lugar, la gestión de las sesiones presenciales, ya que la docencia presentación retransmitida por videoconferencia ofrece un modelo basado en metodologías tradicionales, complicando la aplicación de metodologías activas debido a la dificultad de desarrollar dinámicas que se adapten a participación presencial y online al mismo tiempo. Para intentar paliar las dificultades impuestas por elementos externos a la asignatura, se plantea un enfoque de aula invertida (Bergmann \& Sams, 2012; Kiat \& Kwong, 2014; Moreno-Ruiz et al., 2019). Los vídeos grabados durante las sesiones síncronas del curso 2019-20 se editan y convierten en píldoras de vídeo que se añaden a la batería de píldoras disponibles. Estos vídeos se ponen a disposición del alumnado previamente a la sesión de clase para que durante la sesión se puedan realizar dinámicas y actividades sobre los contenidos teóricos.

En segundo lugar, se decide mantener Microsoft Teams como herramienta central de comunicación y coordinación de la asignatura, de tal forma que el esfuerzo se centra en mejorar la integración de la herramienta en una asignatura que se imparte de forma presencial. La Tabla 1 muestra la adaptación de los principales elementos de la asignatura descritos en el apartado anterior y cómo Microsoft Teams ha tomado mayor peso en gran parte de ellos.

Microsoft Teams se convierte en un elemento central, no solo para la comunicación con el alumnado sino como medio para compartir información, gestionar ciertas dinámicas en las sesiones presenciales o gestionar dudas. A pesar del amplio uso de Teams, es importante aclarar que se sigue manteniendo el espacio en el campus virtual (Figura 2) para mantener la coherencia con el resto de las asignaturas y como herramienta que permite una mejor gestión de la entrega de actividades y la publicación de las calificaciones.
Tabla 1. Evolución de la asignatura durante la crisis sanitaria por COVID-19

\begin{tabular}{|c|c|c|}
\hline Elemento & $\begin{array}{l}\text { Curso 2019-20 con } \\
\text { docencia online }\end{array}$ & $\begin{array}{c}\text { Curso } 2020-21 \text { con } \\
\text { docencia presencial con } \\
\text { asistencia online }\end{array}$ \\
\hline $\begin{array}{l}\text { Campus } \\
\text { virtual }\end{array}$ & $\begin{array}{l}\text { Gestión de contenidos, } \\
\text { entrega de prácticas y } \\
\text { comunicación con el } \\
\text { alumnado. }\end{array}$ & $\begin{array}{l}\text { Se mantiene para la entrega } \\
\text { de prácticas pero se traslada } \\
\text { el espacio principal de } \\
\text { comunicación con el } \\
\text { alumnado a Microsoft } \\
\text { Teams. } \\
\end{array}$ \\
\hline Twitter & $\begin{array}{c}\text { Materiales } \\
\text { complementarios a la } \\
\text { asignatura. }\end{array}$ & $\begin{array}{l}\text { Continúa utilizándose pero } \\
\text { se complementa con un } \\
\text { espacio en Microsoft Teams. }\end{array}$ \\
\hline $\begin{array}{l}\text { Píldoras de } \\
\text { vídeo }\end{array}$ & $\begin{array}{l}\text { Elaboración de píldoras } \\
\text { como materiales de } \\
\text { apoyo a las prácticas. }\end{array}$ & $\begin{array}{c}\text { Adaptación de todas las } \\
\text { grabaciones del curso previo } \\
\text { como materiales centrales } \\
\text { de la asignatura para aplicar } \\
\text { aula invertida. }\end{array}$ \\
\hline Clases & $\begin{array}{c}\text { Sesiones síncronas y } \\
\text { grabaciones de las } \\
\text { sesiones con Blackboard } \\
\text { Collaborate. }\end{array}$ & $\begin{array}{l}\text { Sesiones presenciales con } \\
\text { asistencia online mediante } \\
\text { Blackboard Collaborate y } \\
\text { uso de Microsoft Teams. }\end{array}$ \\
\hline Tutorías & $\begin{array}{l}\text { Incorporación de } \\
\text { Microsoft Teams para } \\
\text { facilitar las tutorías } \\
\text { individuales y grupales, } \\
\text { así como el seguimiento } \\
\text { de la práctica final. }\end{array}$ & $\begin{array}{l}\text { Gestión íntegra de las } \\
\text { tutorías síncronas y } \\
\text { asíncronas así como el } \\
\text { seguimiento de la práctica } \\
\text { final a través de Microsoft } \\
\text { Teams. }\end{array}$ \\
\hline $\begin{array}{l}\text { Defensa de la } \\
\text { práctica final }\end{array}$ & $\begin{array}{c}\text { Videoconferencias a } \\
\text { través de Microsoft } \\
\text { Teams. }\end{array}$ & $\begin{array}{l}\text { Se mantienen las defensas a } \\
\text { través de Microsoft Teams. }\end{array}$ \\
\hline $\begin{array}{l}\text { Interfaces } \\
\text { imaginadas }\end{array}$ & $\begin{array}{c}\text { Integración de Microsoft } \\
\text { Stream, Microsoft Forms } \\
\text { junto con Blackboard } \\
\text { Collaborate. }\end{array}$ & $\begin{array}{l}\text { Uso de Microsoft Stream en } \\
\text { las sesiones presenciales con } \\
\text { participación online a través } \\
\text { de Blackboard Collaborate. }\end{array}$ \\
\hline
\end{tabular}

\section{A. Canales para organizar el espacio}

El espacio de Microsoft Teams se ha organizado a través de la creación de canales, concretamente un canal por cada tema de teoría y uno por cada práctica entregable, de esta forma el material asociado y las dudas están en un mismo espacio, pudiendo enriquecerlo con más materiales, no solo compartidos por el equipo docente sino también por algunos estudiantes.

Además, el equipo se completa con un espacio para la práctica final donde se plantean las dudas, las indicaciones y la información relevante asociada a la misma; y un canal de recursos compartidos donde el alumnado y el equipo docente han compartido contenidos relacionados con la asignatura, sin necesidad de ser materiales educativos como tal.

Por último, a nivel privado, el equipo docente mantiene un canal de coordinación a través del cuál coordinan que todos los grupos reciban las mismas indicaciones y pautas en el aula, ya que en el campus virtual y en Microsoft Teams todos los grupos de la asignatura están juntos.

\section{B. Gestión del alumnado}

Este proceso se lleva a cabo al comienzo de la asignatura y requiere dedicarle algo de tiempo. Existen dos formas de añadir miembros a un equipo en Microsoft Teams: que el equipo docente los añada uno a uno conocimiento su nombre y/o email; o que el estudiante se una a través de un enlace. 


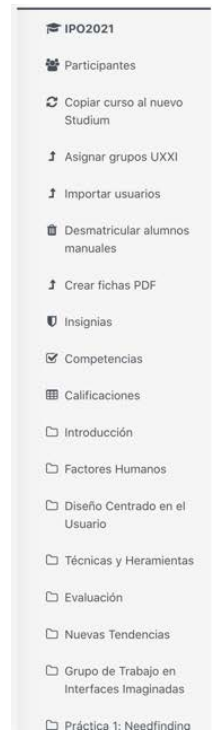

Interacción Persona Ordenador

Grado en Ingeniería Informática

Facultad de Ciencias
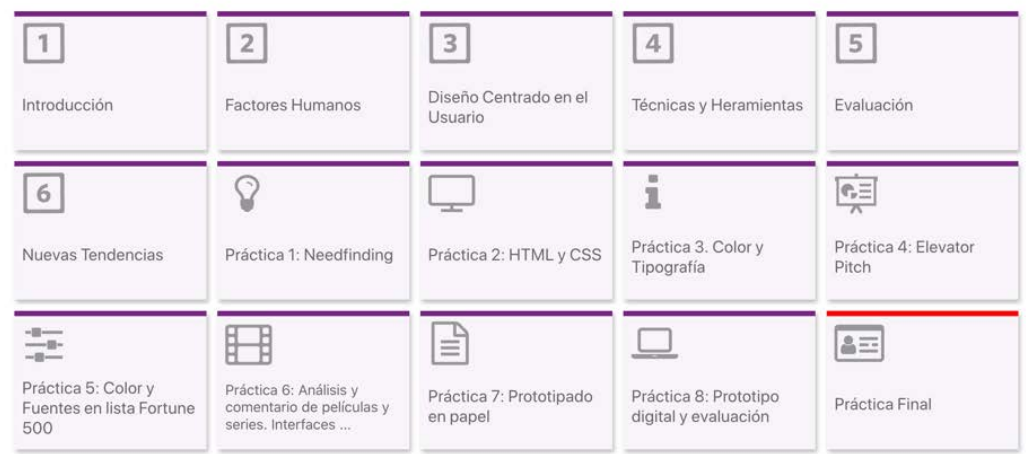

Figura 2. Espacio de la asignatura en el campus virtual durante el curso 2020-21

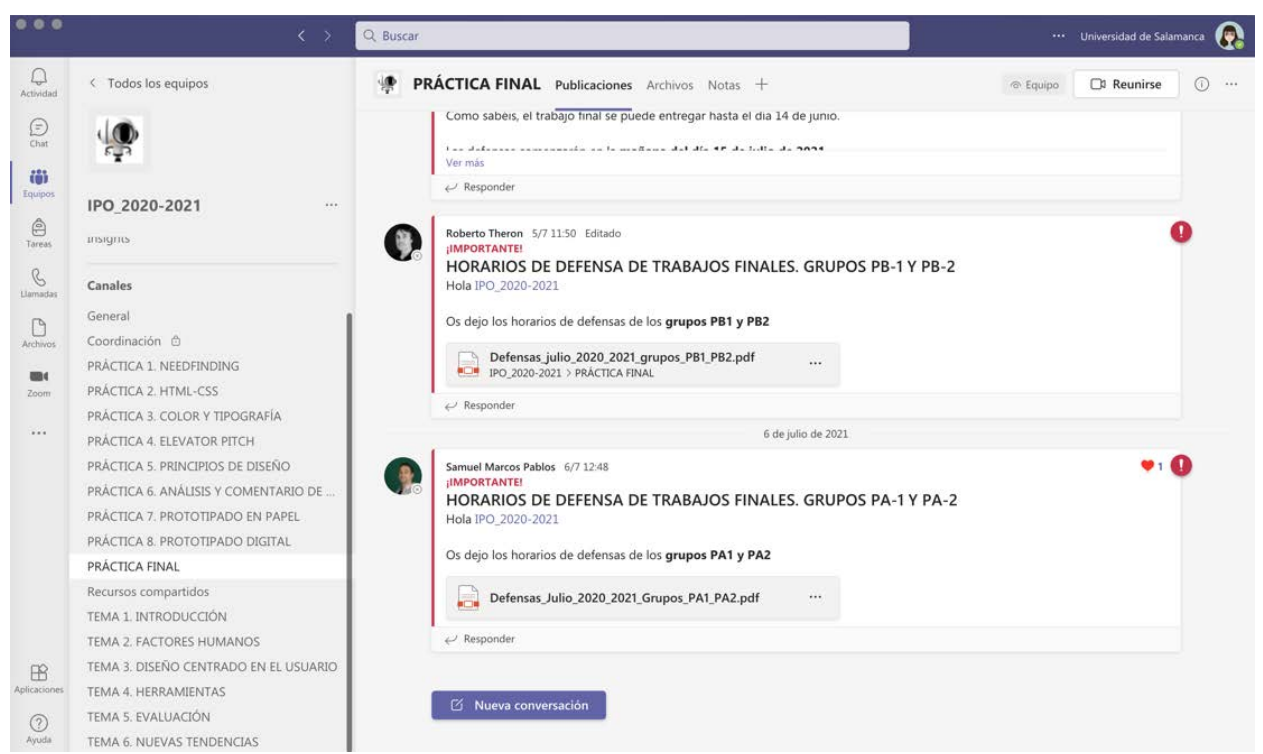

Figura 3. Equipo en Microsoft Teams para el curso 2020-21

En el caso de la Universidad de Salamanca la primera opción no es factible ya que la integración de la cuenta institucional con la cuenta de Microsoft Office no es totalmente operativa, por lo que no todos los estudiantes aparecen inicialmente y no siempre aparecen con nombre y apellidos. Por tanto, el proceso de gestión del alumnado se inicia compartiendo el enlace para que se unan al equipo, de tal forma que el equipo docente debe aceptar las solicitudes de unirse.

En el proceso de gestión de solicitudes cada estudiante es etiquetado para facilitar su identificación y permitir la comunicación con un conjunto de estudiantes concreto (Figura 4). Concretamente se les asignan las siguientes etiquetas:

- Grupo de teoría: TA, TB.

- Subgrupo de prácticas: PA1-1, PA1-2, PA2-1, PA22, PB1-1, PB1-2, PB2-1, PB2-2.

- Grupo de trabajo de prácticas: PA1-GR1, PA1GR2, PA1-GR3...

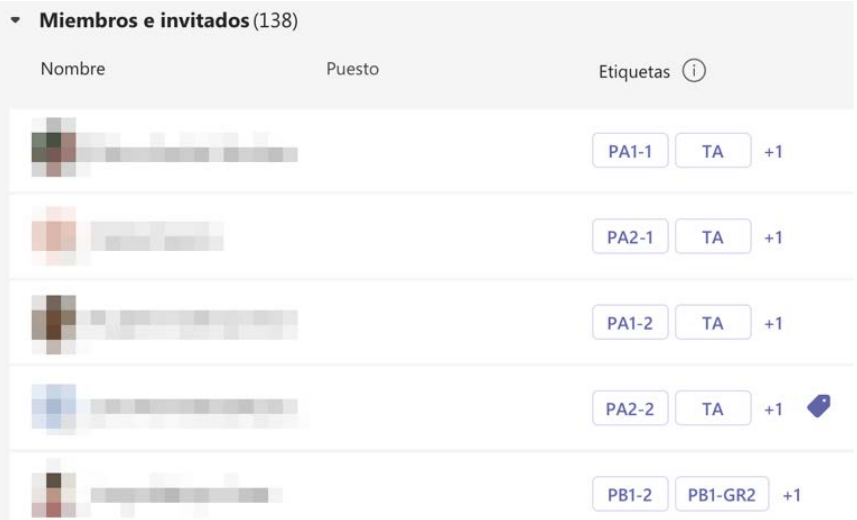

Figura 4. Etiquetas de los miembros del equipo en Microsoft Teams 


\section{Gestión de trabajo en equipo}

Uno de los pilares de la asignatura es la realización de una serie de proyectos que se hacen de forma individual y en grupo. Microsoft Teams proporciona un espacio idóneo para el trabajo en grupo.

Para gestionar el uso de la herramienta por parte del alumnado se realizó una sesión inicial de prácticas en la que una de las tareas, tanto para las personas que estaban en presencial como las que estaban en online, consistía en organizarse en equipos de trabajo utilizando un documento compartido editable a través de Microsoft Teams. En este documento, dentro de una misma clase, debían organizarse en grupos de cinco personas y luego crear una conversación grupal en Teams $\mathrm{y}$ añadir al docente. El principal objetivo era que toda la coordinación del equipo se desarrollara a través de la herramienta y que el docente pudiera responder dudas concretas al equipo dentro de su propio espacio, así como tener información suficiente en caso de que hubiera un problema entre los miembros del equipo.

\section{Integración con otras herramientas}

Por último, destacar la integración de Microsoft Teams con otras herramientas. Si bien no existe una fuerte integración con el ecosistema tecnológico institucional, la herramienta permite el uso de otras herramientas de la suite de Microsoft tales como Microsoft Forms o Microsoft Stream.

En particular, se ha hecho uso de Microsoft Stream para gestionar todos los vídeos de la asignatura, tanto los creados por el equipo docente como los vídeos realizados en prácticas por los estudiantes (Figura 5). Existen muchas otras opciones para crear canales de vídeo, pero la ventaja de Microsoft Stream es la integración con Microsoft Teams, de tal forma que se facilita el acceso a los materiales multimedia y se unifica todo en un mismo espacio. Además de que el contenido no es público, solo visible para los miembros del equipo, esto es, docentes y estudiantes de la asignatura.
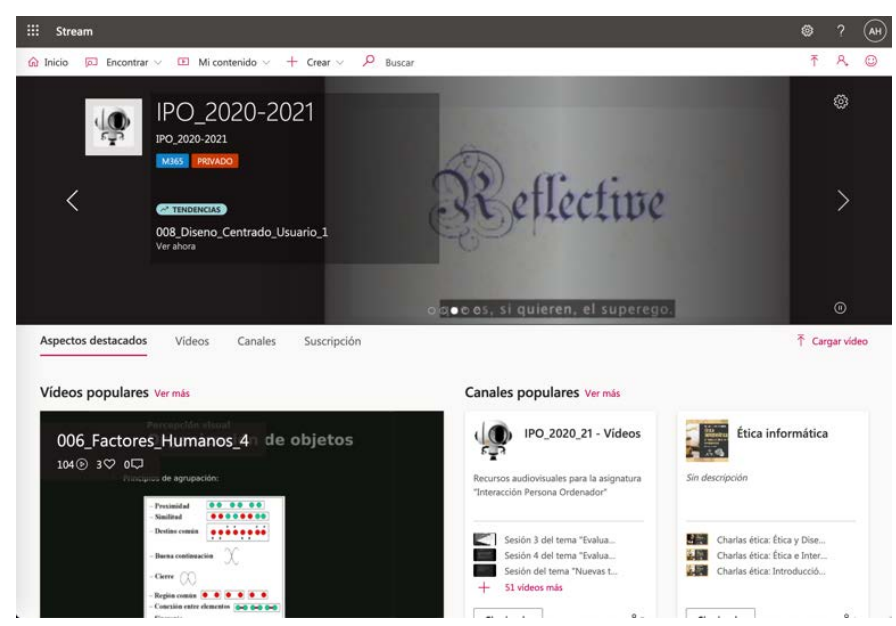

Figura 5. Grupo en Microsoft Stream de la asignatura

\section{RESUltados}

El resultado de incorporar Microsoft Teams como herramienta central de la asignatura es una mejora directa en los procesos de comunicación entre equipo docente y alumnado. Si bien el campus virtual proporciona mecanismos de comunicación, nunca se había logrado que el alumnado utilizara el principal medio de interacción, el foro. Microsoft Teams se acerca más a la forma de comunicarse que tenemos en la actualidad, con mensajes cortos, rápidos, en forma de conversación. Además, aunque Moodle, la herramienta en la que se basa el campus virtual de la USAL, dispone de aplicación móvil, la interacción con la misma no es tan fluida. En cambio, la aplicación de Microsoft Teams ofrece ese formato de conversación rápida, con notificaciones $\mathrm{y}$ conversaciones privadas.

El número de mensajes publicados en los foros del campus virtual ha sido 0 en los últimos cursos académicos. La mayor parte de la comunicación se realizaba en presencial o por email en algunos casos. Desde la incorporación de Microsoft Teams, sobre todo durante el curso 2020-21, se ha reducido casi a 0 la comunicación por email.

Además, otro cambio positivo a la hora de introducir esta herramienta ha sido la transición de tutorías totalmente síncronas y presenciales, a tutorías asíncronas, donde se van resolviendo problemas y dudas en una conversación fluida que se da entre docente y estudiante a lo largo de un periodo de tiempo, sin necesidad por ninguna de las partes de responder en el mismo momento en el que se recibe el mensaje.

Por otro lado, también se han detectado una serie de inconvenientes. En primer lugar, en relación con la comunicación con el alumnado, se presupone una disponibilidad 24/7 del equipo docente. Se trata de una problemática que se debe abordar estableciendo unas normas o pautas que ayuden a mantener un equilibrio entre la rigidez de un horario de tutorías reducido a unas horas y días concretos y la disponibilidad a cualquier hora y día de la semana.

En segundo lugar, la incorporación de Microsoft Teams ha supuesto mantener dos espacios educativos para una misma asignatura. Si bien el espacio del campus virtual únicamente contiene los materiales docentes oficiales y los espacios de entrega de prácticas (Figura 2), todos estos materiales se ponen también a disposición del alumnado en Microsoft Teams para crear los canales para cada tema de teoría y así tener un espacio donde ir compartiendo más materiales adicionales y resolver dudas sobre el temario.

Además, a nivel organizativo e institucional Studium sigue siendo el espacio oficial, por lo que los mensajes oficiales, tales como convocatorias de exámenes o fechas de entrega, deben publicarse en el campus de manera formal y luego en Microsoft Teams porque es donde realmente están pendientes el grueso de los estudiantes.

Finalmente, es importante destacar el uso de la herramienta por parte del alumnado. Una parte de la asignatura se sustenta en el trabajo en equipo, de tal forma que varios estudiantes deben colaborar para realizar algunas de las prácticas. El trabajo en equipo supone un reto a la hora de evitar que haya estudiantes que no trabajen. El uso de Microsoft Teams, si bien no ha resuelto completamente este problema, sí que ha servido como herramienta para aquellos grupos en los que algún miembro no trabajaba, de tal forma que ese grupo al ver estas problemáticas pasaba toda su comunicación para coordinarse al espacio de Microsoft Teams, de tal forma que si el problema continuaba tenían un registro que el equipo docente podía consultar para ayudar a buscar una solución que no perjudicase a las personas que sí trabajaban. 


\section{CONCLUSIONES}

El presente trabajo describe como se ha integrado Microsoft Teams en la docencia de la asignatura Interacción PersonaOrdenador, como ejemplo real para integrar este tipo de herramientas en otras asignaturas, independientemente de si la docencia se desarrolla en formato presencial, online o híbrido.

Existen un gran número de herramientas similares a Microsoft Teams que se utilizan en el ámbito laboral para la gestión de equipos y la comunicación tanto síncrona como asíncrona, siendo Slack una de las más extendidas. La decisión de utilizar Teams no radica en un análisis de su funcionalidad sino en una cuestión práctica, la integración en el ecosistema tecnológico institucional, a pesar de las diferentes carencias relacionadas con la experiencia de usuario que se han detectado a lo largo de su uso, tanto por el equipo docente como por el propio alumnado.

En cuanto a líneas futuras de trabajo, el uso de Microsoft Teams seguirá estando presente como herramienta central de la asignatura, si bien se buscará resolver los principales problemas detectados. Por un lado, la gestión de la disponibilidad por parte del profesorado, y por otro lado incrementar el uso por parte del alumnado para gestionar su trabajo en equipo.

\section{AGRADECIMIENTOS}

Este trabajo tiene el apoyo del proyecto de innovación docente "Diseño y creación de un SPOC sobre consideraciones éticas en el desarrollo software para los estudiantes del Grado en Ingeniería Informática" (ID2020/002) financiado por la Universidad de Salamanca (España) en el curso 2020-21.

\section{REFERENCIAS}

Abella García, V., Grande de Prado, M., García-Peñalvo, F. J., \& Corell, A. (2020). Guía de recomendaciones para la evaluación online en las Universidades Públicas de Castilla y León. Versión 1.1. https://bit.ly/2SqTtR2

Bergmann, J., \& Sams, A. (2012). Flip your classroom: Reach every student in every class every day. International Society for Technology in Education.

Estruch, V., \& Silva, J. (2006). Aprendizaje basado en proyectos en la carrera de Ingeniería Informática Actas de las XII Jornadas de la Enseñanza Universitaria de la Informática, JENUI 2006, Deusto, Bilbao.

Fardoun, H., González-González, C. S., Collazos, C. A., \& Yousef, M. (2020). Estudio exploratorio en Iberoamérica sobre procesos de enseñanza-aprendizaje y propuesta de evaluación en tiempos de pandemia. Education in the Knowledge Society 21. doi:10.14201/eks.23437

García-Peñalvo, F. J. (2020). Jurassic World: El mundo online caído Jornadas Vir UVa les, Valladolid, España. https://bit.ly/2OGAJdX

García-Peñalvo, F. J., \& Corell, A. (2020). La CoVId-19: ¿enzima de la transformación digital de la docencia o reflejo de una crisis metodológica y competencial en la educación superior? CampusVirtuales, 9(2), 83-98.

García-Peñalvo, F. J., Corell, A., Abella-García, V., \& Grande, M. (2020). La evaluación online en la educación superior en tiempos de la COVID-19. Education in the Knowledge Society, 21. doi:10.14201/eks.23086

García-Peñalvo, F. J., Corell, A., Rivero-Ortega, R., RodríguezConde, M. J., \& Rodríguez-García, N. (2021). Impact of the COVID-19 on Higher Education: An ExperienceBased Approach. En F. J. García-Peñalvo (Ed.), Information Technology Trends for a Global and Interdisciplinary Research Community (pp. 1-18). IGI Global.

García-Peñalvo, F. J., García-Holgado, A., Vázquez-Ingelmo, A., \& Sánchez Prieto, J. C. (2021). Planning, communication and active methodologies: Online assessment of the software engineering subject during the COVID-19 crisis. RIED. Revista iberoamericana de educación a distancia, 24(2), 41-66. doi:10.5944/ried.24.2.27689

Hernández-Ramos, J. P., Martínez-Abad, F., \& Sánchez-Prieto, J. C. (2021). El empleo de videotutoriales en la era post COVID19: valoración e influencia en la identidad docente del futuro profesional. Revista de Educación a Distancia (RED), 21(65). doi:10.6018/red.449321

Kiat, P. N., \& Kwong, Y. T. (2014, 23-25 April 2014). The flipped classroom experience. 2014 IEEE 27th Conference on Software Engineering Education and Training (CSEE\&T),

Llorens-Largo, F. (2020). Docencia de emergencia: cómo cambiar el motor en pleno vuelo. Universídad. Recuperado el 15 Jul. de https://bit.ly/3cpHVEV

Llorens-Largo, F., Villagrá-Arnedo, C., Gallego-Durán, F., \& Molina-Carmona, R. (2021). COVID-proof: cómo el aprendizaje basado en proyectos ha soportado el confinamiento. Campus Virtuales, 10(1), 73-88.

Macias, J. A. (2012). Enhancing Project-Based Learning in Software Engineering Lab Teaching Through an EPortfolio Approach. IEEE Transactions on Education, 55(4), 502-507. doi:10.1109/TE.2012.2191787

Moreno-Ruiz, L., Castellanos-Nieves, D., Braileanu, B. P., González-González, E. J., Sánchez-De La Rosa, J. L., Groenwald, C. L. O., \& González-González, C. S. (2019). Combining Flipped Classroom, Project-Based Learning, and Formative Assessment Strategies in EngineeringStudies. International Journal of Engineering Education, 35(6(A)), 1673-1683.

Therón, R., Cruz-Benito, J., García-Sánchez, F., Santamaria, R., \& García-Peñalvo, F. J. (2017). Innovación en la enseñanza de la Interacción Persona-Ordenador: interfaces imaginadas, ciencia-ficción y trabajo con usuarios reales. En M. L. Sein-Echaluce Lacleta, Á. Fidalgo Blanco, \& F. J. García-Peñalvo (Eds.), IV Congreso Internacional sobre Aprendizaje, Innovación y Competitividad-CINAIC (Zaragoza 4-6 Octubre 2017) (pp. 480-485). Universidad de Zaragoza. doi:10.26754/CINAIC.2017.000001_100

Zubillaga, A., \& Gortazar, L. (2020). COVID-19 y educación: Problemas, respuestas y escenarios. https://bit.ly/3auXnP8 